\title{
Vaccination remains the Mainstay of Prevention Strategy of Japanese Encephalitis
}

\section{Mahendra Pal ${ }^{\star}$ Gujarat, India \\ Article Info \\ *Corresponding author: Prof. Dr. Mahendra Pal \\ Founder of Narayan Consultancy on Veterinary Public Health and Microbiology 4 Aangan, Jagnath Ganesh Dairy Road Anand-388001, Gujarat, India \\ E-mail: palmahendra2@gmail.com}

Founder of Narayan Consultancy on Veterinary Public Health and Microbiology, 4 Aangan, Jagnath Ganesh Dairy Road, Anand-388001,

\section{Received: October 11, 2018 \\ Accepted: November 1, 2018 \\ Published: January 2, 2019}

Citation: Pal M. Vaccination remains the Mainstay of Prevention Strategy of Japanese Encephalitis. Madridge J Vaccines. 2019; 3(1): 64-65

doi: $10.18689 / \mathrm{mjv}-1000114$

Copyright: @ 2019 The Author(s). This work is licensed under a Creative Commons Attribution 4.0 International License, which permits unrestricted use, distribution, and reproduction in any medium, provided the original work is properly cited.

Published by Madridge Publishers

\section{Keywords: Vaccination; Smallpox; Immunization; Japanese encephalitis; Zika virus.}

Vaccination (Immunization) plays a pivotal role to protect the health of humans and animals by preventing and controlling many infectious diseases, which are prevalent in developing as well as in developed countries of the world. Immunization is considered as the most successful and cost-effective public health interventions throughout the world. The eradication of smallpox and elimination of measles and poliomyelitis in regions of the world was possible because of immunization programs. Moreover, vaccination also resulted in substantial reductions of the morbidity and mortality attributed to diphtheria, pertussis and tetanus. It is important to mention that immunization saves up to three million people from infectious diseases every year.

There are many vector borne viral zoonoses, such as chikungunya fever, Crimean Congo haemorrhagic fever, dengue fever, Eastern equine encephalitis, Ganjam virus disease, ilheus fever Japanese encephalitis, Kyasanur forest disease, Mayaro fever, Murray Valley encephalitis, Phlebotomus fever, Rift Valley fever, Rossa river fever, Saint Louis encephalitis, Sindbis fever, Venezuekan equine encephalitis, yellow fever, Western equine encephalitis and West Nile fever which cause high morbidity and mortality worldwide. Among this Japanese encephalitis (JE) also known as 'brain fever' is an important vector borne zoonotic disease of major public health concern particularly to Asian countries.

Japanese encephalitis is an emerging and re-emerging metazoonosis, which was first time described in 1871 from Japan. Since then the disease has been recorded from many nations of the world including Australia, Bangladesh, China, India, Indonesia, Kampuchea, Korea, Laos, Malaysia, Myanmar, Nepal, Pakistan, Philippines, Singapore, Sri Lanka, Taiwan and Thailand. The disease is caused by Japanese encephalitis virus (single stranded RNA) which belongs to the same family (Flaviviridae) as dengue virus, yellow fever virus and Zika virus. It is transmitted by the bite of infected Culex mosquitoes, mainly Culex tritaeniorhynchus which usually breeds in rice fields. It is stated that Japanese encephalitis virus commonly lives in natural reservoirs like pigs and wading birds; therefore complete eradication of disease seems to be impracticable.

The majority of Japanese encephalitis cases in endemic countries are encountered in children younger than 15 years, as adults are often already immune to the disease. Further, males were more frequently affected than females. It is an important public health threat, as case fatality rates may reach up to $30 \%$ and long-term neuropsychological sequelae are observed in $30-50 \%$ of its survivors. A major outbreak of Japanese encephalitis in 2005 mainly confined to Gorakhpur, Uttar Pradesh (India) affected 6,061 persons and caused 1,501 deaths.

Due to the absence of treatment for Japanese encephalitis and recent expansion of the geographic range of this devastating disease, the World Health Organization has 
acknowledged the necessity of improved surveillance for the disease and also emphasized the incorporation of Japanese encephalitis vaccine into routine immunization program wherever the disease remains a major public health threat. In addition to immunization, vector control, environmental sanitation and use of protective clothing and mosquitoes repellents are also crucial to eradicate this serious viral disease. It is pertinent to mention that presently vaccination is considered the single, the most important measure for the control of Japanese encephalitis, which is the major viral metazoonosis of global significance.

Luckily, a vaccine is available to prevent the spread of Japanese encephalitis in humans. Immunization Program in India was introduced in 1978 as Expanded Program of Immunization. As the program gained momentum in 1985, it was expanded as Universal Immunization Program to cover all districts in the country by 1989-90. In India, live attenuated SA-14-14-2 vaccine against Japanese encephalitis was introduced in the routine immunization under Universal Immunization Program in the 181 endemic districts of the country. Recently the Government of India has announced the introduction of one dose of Japanese encephalitis vaccine for adults in endemic districts. SA $14-14-2$ is a live-attenuated vaccine that is manufactured by the Chengdu Institute of Biological Products, China and is certified for use in China since 1988. Till date over 400 million doses have been administered in China and other Asian countries with a remarkable safety record. In recent times this vaccine has been approved for use in other countries such as India, Nepal, South Korea, Sri Lanka and Thailand.
In India, the imported SA 14-14-2 vaccine has been used to immunize over 9.3 million children aged between 1 and 15 years during the summer of 2006 in endemic states like Assam, Bihar, Jharkhand, Uttar Pradesh and West Bengal. In addition, two vaccines, namely JEEV (a purified inactivated (killed) vaccine that uses the SA 14-14-2 strain of Japanese encephalitis virus, and is manufactured by Biological E Ltd., Hyderabad, India; and JENVAC (an inactivated (killed) Verocell derived vaccine prepared from an Indian strain of Japanese encephalitis virus, is manufactured by Bharat Biotech International Ltd., Hyderabad, India). Both Indian manufactured vaccines are expensive as compared to SA 14-14-2 vaccine. As live attenuated SA 14-14-2 vaccine is cheaper than two Indian vaccines, hence it can be recommended for mass vaccination against Japanese encephalitis. It is stated that Japanese encephalitis vaccine can be simultaneously taken with other vaccines.

The persons travelling to endemic areas are advised to take Japanese encephalitis vaccination to protect from this vector borne disease. It is emphasized that all endemic countries should integrate Japanese encephalitis vaccine into the national immunization program in order to protect the health of children under 15 years of age from this life threatening viral zoonosis. There is a need to create more awareness among the public that Japanese encephalitis vaccine is much safer than getting the disease.

The author wishes to dedicate this paper in the memory of Edward Jenner (English Scientist) and Louis Pasteur (French Scientist) who were the pioneers in the field of vaccination and vaccine. 